\title{
Utility of olfactory identification test for screening of cognitive dysfunction in community-dwelling older adults
}

\author{
Satoshi Nogi ${ }^{1}$, Kentaro Uchida ${ }^{\text {Corresp., } 1}{ }^{\text {, Jumpei Maruta }}{ }^{1,2}$, Hideo Kurozumi ${ }^{1}$, Satoshi Akada ${ }^{1}$, Masatsugu Shiba ${ }^{3}$, \\ Koki Inoue ${ }^{1,4}$ \\ ${ }^{1}$ Neuropsychiatry, Osaka City University Graduate School of Medicine, Osaka, Osaka, Japan \\ 2 Medical Center for Dementia, Osaka City Kosaiin Hospital, Osaka, Osaka, Japan \\ 3 Medical statistics, Osaka City University Graduate School of Medicine and Faculty of Medicine, Osaka, Osaka, Japan \\ ${ }^{4}$ Center for Brain Science, Osaka City University Graduate School of Medicine and Faculty of Medicine, Osaka, Osaka, Japan \\ Corresponding Author: Kentaro Uchida \\ Email address: uchida.kentaro@med.osaka-cu.ac.jp
}

Background. There is a need for a large-scale screening test that can be used to detect dementia in older individuals at an early stage. Olfactory identification deficits have been shown to occur in the early stages of dementia, indicating their usefulness in screening tests. This study investigated the utility of an olfactory identification test as a screening test for mild cognitive dysfunction in community-dwelling older people. Methods. The subjects were city-dwelling individuals aged over 65 years but under 85 years who had not been diagnosed with dementia or mild cognitive impairment. The Japanese version of the Mild Cognitive Impairment Screen was used to evaluate cognitive function. Based on the results, the subjects were divided into two groups: healthy group and cognitively impaired group. Olfactory identification abilities based on the Japanese version of the University of Pennsylvania Smell Identification Test were compared between the groups. Results. There were 182 participants in total: 77 in the healthy group and 105 in the cognitively impaired group. The mean olfactory identification test score of the cognitively impaired group was significantly lower than that of the healthy group. The cognitive impairment test score was significantly correlated with the olfactory identification test score. Conclusions. Cross-sectional olfactory identification deficits at baseline in community-dwelling older adults reflected cognitive dysfunction. Assessing olfactory identification ability might be useful as a screening test for mild cognitive dysfunction in community-dwelling older people. 


\section{Utility of olfactory identification test for screening of}

\section{2 cognitive dysfunction in community-dwelling older adults}

3

4 Satoshi Nogi ${ }^{1}$, Kentaro Uchida ${ }^{1}$, Jumpei Maruta ${ }^{1,2}$, Hideo Kurozumi ${ }^{1}$, Satoshi Akada ${ }^{1}$,

5 Masatsugu Shiba ${ }^{3}$, Koki Inoue ${ }^{1,4}$

6

$7{ }^{1}$ Department of Neuropsychiatry, Osaka City University Graduate School of Medicine, Osaka,

8 Japan

$9{ }^{2}$ Medical Center for Dementia, Osaka City Kosaiin Hospital, Osaka, Japan

$10{ }^{3}$ Medical statistics, Osaka City University Graduate School of Medicine and Faculty of

11 Medicine, Osaka, Japan

$12{ }^{4}$ Center for Brain Science, Osaka City University Graduate School of Medicine and Faculty of

13 Medicine, Osaka, Japan

14

15 Corresponding author:

16 Kentaro Uchida M.D., Ph.D.

17 Department of Neuropsychiatry, Osaka City University Graduate School of Medicine, Osaka, 18 Japan

19 1-4-3, Asahimachi, Abeno-ku, Osaka city, Osaka, Japan

20 Email address: uchida.kentaro@med.osaka-cu.ac.jp 


\section{Abstract}

23 Background. There is a need for a large-scale screening test that can be used to detect dementia

24 in older individuals at an early stage. Olfactory identification deficits have been shown to occur

25 in the early stages of dementia, indicating their usefulness in screening tests. This study

26 investigated the utility of an olfactory identification test as a screening test for mild cognitive

27 dysfunction in community-dwelling older people.

28 Methods. The subjects were city-dwelling individuals aged over 65 years but under 85 years

29 who had not been diagnosed with dementia or mild cognitive impairment. The Japanese version

30 of the Mild Cognitive Impairment Screen was used to evaluate cognitive function. Based on the

31 results, the subjects were divided into two groups: healthy group and cognitively impaired group.

32 Olfactory identification abilities based on the Japanese version of the University of Pennsylvania

33 Smell Identification Test were compared between the groups.

34 Results. There were 182 participants in total: 77 in the healthy group and 105 in the cognitively

35 impaired group. The mean olfactory identification test score of the cognitively impaired group

36 was significantly lower than that of the healthy group. The cognitive impairment test score was

37 significantly correlated with the olfactory identification test score.

38 Conclusions. Cross-sectional olfactory identification deficits at baseline in community-dwelling

39 older adults reflected cognitive dysfunction. Assessing olfactory identification ability might be

40 useful as a screening test for mild cognitive dysfunction in community-dwelling older people. 


\section{Introduction}

43 The number of people with dementia worldwide is expected to rise to 74.7 million by 2030 . The

44 Asian region, including Japan, accounts for the largest number of newly diagnosed dementia 45 cases, at 4.9 million or $49 \%$ of the global total. ${ }^{1}$ Early treatment and support can help delay the 46 progression of dementia, ${ }^{2}$ thus it is essential that the disease is detected at an early stage. It has

47 been reported that the severity of dementia is moderate at the initial diagnosis in many cases; ${ }^{3}$

48 therefore, screening at an early stage is an important requirement.

49 Traditional dementia tests, such as the Mini-Mental State Examination (MMSE), and mild 50 cognitive impairment (MCI) tests, such as the Montreal Cognitive Assessment and Rivermead

51 Behavioral Memory Test, are used to evaluate cognitive function. However, these tests have

52 drawbacks, such as the time, the inspector and the expertise of psychologists required for testing.

53 Hence, there is a need for screening tests that are simple for the users and easy to administer to

54 large numbers of older people.

55 In many types of age-related dementia, such as Alzheimer's disease (AD), Lewy body diseases

56 (LBD), cerebrovascular dementia, and frontotemporal lobe dementia, olfactory disturbances have

57 been found to occur in the early stages of the disease. ${ }^{4}$ Wilson et al. reported that, cross-

58 sectionally, olfactory identification is significantly related to the incidence of MCI. ${ }^{5}$ Roalf et al.

59 demonstrated the presence of olfactory identification deficits in patients with MCI, ${ }^{6}$ while

60 Windon et al. showed that olfactory deficits could predict progression from cognitively normal

61 function to MCI and apparently precede cognitive dysfunction in some patients. ${ }^{7}$ Recently, the

62 relationship between cognitive dysfunction and olfactory function deficits in Japanese diagnosed

63 with MCI has also been reported. ${ }^{8}$ 
64 Olfactory identification tests are easy to use, non-invasive, and can be performed in less time

65 than traditional tests; they are unaffected by hearing and visual impairments, which increase with

66 age and often affect other forms of testing. Thus, olfactory identification tests are thus among the

67 candidate screening tests for cognitive dysfunction and can be incorporated into health checkups.

68 Several studies have shown an association between olfactory identification and cognitive

69 function in community-dwelling older adults. ${ }^{9,10}$ However, olfactory disorders have been shown

70 to have genetic and cultural disparities, ${ }^{11}$ and, therefore, need to be investigated in each genetic

71 or cultural background. To our best knowledge, the utility of such tests as screening tools for

72 mild cognitive dysfunction among community-dwelling older people in Japan has not been

73 demonstrated.

74 We aimed to investigate the relationship between olfactory identification deficits and cognitive

75 function in community-dwelling older people. Additionally, we sought to evaluate the utility of

76 such a test as a screening tool for mild cognitive dysfunction in older individuals.

\section{Materials \& Methods}

\section{Participants}

80 The participants were people over 65 years and under 85 years of age, living in a city in the

81 Kansai region of Japan. They were recruited from among participants of health measurement

82 events and other similar events held in the city between October and December 2019 by Coomin

83 Corp, a private company funded by a local administrative organ that runs health promotion

84 projects.

85 The exclusion criteria were as follows: current diagnosis of dementia or MCI, severe mental

86 illness, nasal diseases that might affect olfaction, history of head injury, brain tumor, 
87 cerebrovascular disorder, or taking drugs that may affect olfaction.

88 This study was approved by the Ethical Committee of Osaka City University Graduate School of

89 Medicine (No.4428). All eligible participants were informed of the purposes and methods of the

90 study, and in accordance with the Declaration of Helsinki, written informed consent was

91 obtained from all participants before including them in the study. They were considered to have

92 sufficient capacity to give consent because they were voluntarily participating in these events

93 and were able to independently perform activities of daily living. In addition, we used plain

94 language in the explanations to ensure sufficient understanding, and paused the examination if

95 the participants wished, so as not to be a burden to them.

\section{Cognitive function}

98 The Mild Cognitive Impairment Screen (MCIS) is a cognitive function assessment scale derived

99 from the Consortium to Establish a Registry for Alzheimer's Diseases (CERAD) 10-word List

100 Learning Test of the National Institute of Aging, which forms part of the Consortium's

101 neuropsychological battery. In previous studies, the MCIS has been found to be useful for

102 distinguishing between normal cognitive function and MCI, using the Clinical Dementia Rating,

103 based on the patient's and caregiver's complaints, and has been reported to distinguish normal

104 cognitive function from MCI with 94\% sensitivity and 97\% specificity. ${ }^{12,13}$ The Japanese version

105 of the MCIS (JMCIS) has the same performance as the MCIS. ${ }^{14}$ Cognitive function was assessed

106 using the JMCIS and MMSE. We used the official Japanese version of the MMSE test form

107 prepared under contract with the original publisher (Psychological Assessment Resources, Inc.)

108 for each participant in this study.

109 The results of JMCIS were reported electronically, and the patient's memory capabilities were 
110 calculated using correspondence analysis and were reported as a score called the memory

111 performance index (MPI), which took into account sex, age, and education history. The score

112 was expressed as a number from 0 to 100 , with 50.2-100 indicating normal cognitive function

113 and $0-49.8$ indicating cognitive impairment. In about $1 \%$ of cases, the score was between 49.8

114 and 50.2, where a judgment could not be made, in which case the MPI was judged as borderline

$115(49.8<$ MPI $<50.2)$.

116 The participants were divided into groups with normal cognitive function (healthy group; $50.2 \leq$

$117 \mathrm{MPI} \leq 100$ ) or impaired cognitive function (cognitively impaired group; $0 \leq \mathrm{MPI} \leq 49.8$ ). In this

118 study, we included older adults who were voluntarily participate in events and were able to

119 independently perform activities of daily living, and we considered that the cognitive impaired

120 group had mild cognitive dysfunction. The MMSE was used as a criterion to recommend a

121 hospital visit, and participants with scores $\leq 23$, used as a cutoff value, ${ }^{15}$ were recommended to

122 seek medical attention.

123

\section{Olfactory identification ability}

125 The olfactory identification ability was assessed using the Japanese version of the University of

126 Pennsylvania Smell Identification Test (UPSIT-J). The UPSIT was developed by the University

127 of Pennsylvania; ${ }^{16}$ it is the most commonly used olfactory identification test worldwide.

128 Identification deficits in the UPSIT have been reported to be increased (low UPSIT score) in

129 patients with AD and Parkinson's disease (PD) ${ }^{17}$. This test has been shown to be useful as a

130 screening tool for amnesic symptoms in patients with $\mathrm{AD}^{18}$. Moreover, the utility of the

131 Japanese version has been demonstrated ${ }^{19}$. The UPSIT is a 40 -item scratch-and-sniff odorant-

132 identification forced-choice test. In this study, 20 odorants (pizza, bubble gum, mint, banana, 
133 sandalwood, onion, baby powder, cinnamon, gasoline, cedar, daffodil, rubber tire, pickle,

134 popcorn, orange, wintergreen, garlic, grass, soap, and rose) were tested to shorten and simplify

135 the test. These 20 items were selected to avoid deviating from the percentage of correct answers,

136 with reference to the percentage of correct answers in people with normal olfaction ${ }^{19}$. The total

137 score was used as the olfactory score.

138

139 Awareness of function/dysfunction

140 A questionnaire was administered to the participants to determine whether memory loss and

141 olfactory deficit were accurately recognized by community-dwelling older individuals.

142

143 Statistical analysis

144 Statistical analyses were performed using EZR (Saitama Medical Center, Jichi Medical

145 University, Saitama, Japan), which is a graphical user interface for R (The R Foundation for

146 Statistical Computing, Vienna, Austria, version 2.13.0). After performing normality tests, the

147 Mann-Whitney $U$ test was used to compare the average data of non-parametric values (UPSIT-J,

148 MPI, MMSE scores). Age was compared using an unpaired Student's $t$-test. Awareness was

149 analyzed using a chi-square test. The correlations between UPSIT-J and MPI were assessed

150 using Spearman's rank correlation coefficient. P values $<0.05$ were considered statistically

151 significant.

152

153 Results

154 Cohort demographics 
155 A total of 195 participants (31 men and 164 women) were recruited. Eight participants who had

156 difficulty continuing the test until the end, four participants who had a history that interfered with

157 their ability to perform the test, and one participant who was judged to have borderline MPI as

158 per the JMCIS were excluded, resulting in the final number of 182 participants $(28$ men, 154

159 women; age, $76.5 \pm 4.7$ years).

160 According to the MPI score from the JMCIS, the participants $(\mathrm{n}=77)$ with scores of 50.2-100

161 (13 males, 64 females; age $73.5 \pm 4.3$ years; MPI 57.00, range $=50.25-74.70)$ were assigned to

162 the healthy group and those $(n=105)$ with scores of $49.8-0(15$ males, 90 females; age $78.7 \pm$

163 3.7; MPI 39.76, range $=15.55-49.62)$, to the cognitively impaired group. The demographic data

164 of the subjects are shown in Table 1.

165 There was a significant difference in the MMSE scores between the healthy and cognitively

166 impaired groups $(\mathrm{p}<0.001)$. There were no subjects with MMSE scores below 23 in the healthy

167 group, but 20 subjects in the cognitively impaired group had MMSE scores $\leq 23$. We

168 recommended these participants seek medical attention.

169

170 UPSIT-J score

171 The UPSIT-J scores for all subjects ranged from 3.00 to 18.00 points, with a median of 13.00

172 (range, 3.00-18.00). The difference in scores between the healthy group (13.00, range

$1737.00-18.00)$ and the cognitively impaired group (12.00, range 3.00-17.00) was significant $(\mathrm{p}=$

174 0.002; Figure 1).

175

176 Correlation between UPSIT-J score and MPI 
177 Figure 2 shows the correlation between the UPSIT-J score and MPI in all patients. There was a

178 significant correlation between the two $(\mathrm{r}=0.377, \mathrm{p}<0.001)$.

180 Awareness of memory loss and olfactory deficits

181 There was no difference between the healthy and cognitively impaired groups in terms of

182 awareness of memory loss and olfactory deficits. In the healthy group, 53 participants $(68.8 \%)$

183 were aware, and $24(31.2 \%)$ were unaware of memory impairment. In the cognitively impaired

184 group, $85(81.0 \%)$ were aware, and $20(19.0 \%)$ were not aware $(\mathrm{p}=0.087)$ of memory

185 impairment. In the healthy group, 22 participants $(28.6 \%)$ were aware, and $55(71.4 \%)$ were

186 unaware of olfactory impairments. In the cognitively impaired group, $28(26.7 \%)$ were aware,

187 and $77(73.3 \%)$ were unaware $(p=0.91)$ of olfactory deficits. The presence or absence of

188 awareness of olfactory deficits did not result in a significant difference in olfactory scores $(\mathrm{p}=$ 189 0.27).

190

\section{Discussion}

192 This study examined the utility of the olfactory identification test as a screening tool for mild

193 cognitive dysfunction in community-dwelling older people in Japan, considering that this test

194 may be appropriate for screening a large number of individuals. Cognitive function and olfactory

195 identification were examined in community-dwelling older people without a diagnosis of

196 dementia or MCI. We found that older people with mild cognitive dysfunction showed olfactory

197 identification deficits. Olfactory identification deficits in community-dwelling older individuals

198 indicate the presence of cognitive dysfunction, reflecting early dementia symptoms, given that 
199 the mean UPSIT-J score of the cognitively impaired group was significantly lower than that of

200 the healthy group, and the scores were also significantly correlated with MPI.

201 Olfactory identification deficits have been reported in many age-related dementia cases with

202 varying degrees and frequencies, and are usually present from disease onset. We defined the 203 cognitively impaired group using the JMCIS score, which sensitively detects MCI, and found

204 that olfactory function was impaired even in patients with relatively mild cognitive dysfunction.

205 This result was consistent with the findings of Wilson et al. ${ }^{5}$

206 Among dementia-causing diseases, olfactory identification deficits in AD and PD patients are

207 reportedly more frequent and more severe than other deficits. Differences in olfactory decline in

$208 \mathrm{AD}$ and PD patients have also been reported..$^{20}$ In both diseases, the abilities related to the three

209 olfaction functions (detection threshold, discrimination, and identification) are reduced, but

210 olfactory identification deficits are more pronounced in AD patients than in PD patients. Thus, it

211 has been suggested that olfactory identification should be emphasized in screening tests for

212 AD. ${ }^{20}$

213 The MPI was derived from responses to items in the JMCIS, namely the three immediate and

214 one delayed recall trials of the 10-word list developed by the National Institute of Aging's

215 CERAD. This index strongly reflects short-term memory impairment, an early AD symptom. ${ }^{12}$

216 In fact, in a previous MCIS study, when patients with MCI were separated into AD and non-AD

217 groups, the sensitivity of the MCIS was higher for the AD group. ${ }^{12}$ Umeda-Kameyama et al.

218 reported that olfactory function mainly reflects recall ability. ${ }^{21}$ Other studies have shown that

219 olfactory deficits are proportional to cognitive impairment in amnestic but not in non-amnestic

220 MCI patients. ${ }^{22}$ These reports suggest that AD patients with early symptoms of short-term

221 memory impairment are likely to develop olfactory identification deficits at an early stage. The 
222 correlation between the MPI, a sensitive indicator of these symptoms, and olfactory

223 identification deficits found in this study is consistent with the findings of previous findings.

224 These findings can be explained by pathological brain changes. Neurogenic fiber changes in the

225 olfactory bulb (OB); higher olfactory pathways; piriform, amygdala, and entorhinal cortices; and

226 secondary olfactory cortex (hippocampus, orbitofrontal cortex), which are responsible for

227 olfactory memory and odor interpretation, are among the earliest pathological features of AD. ${ }^{23}$

228 These brain regions integrate odor information from the olfactory pathway with projection areas

229 that are involved in odor naming and recall. These neurofibrillary changes cause olfactory

230 identification dysfunction. ${ }^{24} \mathrm{~A}$ strong association between olfactory identification deficits and

231 atrophy of memory areas and of the medial temporal lobe has also been reported in Japanese AD

232 patients. ${ }^{25}$ In addition to the OB and primary olfactory cortex, other olfaction-related cortical

233 structures, including the periamygdaloid, piriform, and entorhinal cortices, have been shown to

234 be susceptible to alpha-synuclein pathology in patients with PD or incidental LBD. Of note, the

235 alpha-synuclein pathology does not immediately spread to adjacent cortices that are unrelated to

236 olfactory processing. ${ }^{26}$ Thus, the pathological changes are consistent with the involvement of

237 olfactory identification deficits in early cognitive dysfunction.

238 Olfactory identification in community-dwelling older individuals reflects cognitive dysfunction.

239 Olfactory identification tests in community-dwelling older people are useful tools for screening

240 cognitive dysfunction, and our results suggest that they may be useful as screening tests for MCI

241 and dementia. These results agree with those of Sohrabi et al., who reported the significant

242 association between the cognitive function and olfactory ability in community-dwelling older

243 individuals, ${ }^{27}$ suggesting that olfactory identification tests can identify community-dwelling 
244 older adults with cognitive dysfunction. Sohrabi et al. also supported the inclusion of olfactory

245 assessment in the standard physical examination of older adults. ${ }^{27}$

246 Our study also showed that it is difficult to perceive memory loss and olfactory deficits

247 accurately in daily life. This is because, as a secondary outcome of the present study, we

248 investigated the participants' self-reported awareness of memory loss and olfactory deficits and

249 found that neither of these differed significantly between the healthy and cognitively impaired

250 groups $(\mathrm{p}>0.05)$. Although people with or without cognitive dysfunction were excessively

251 aware of memory loss, many were unaware of olfactory deficits. Murphy et al. also reported that

252 the prevalence identified by self-reports of olfactory deficits was significantly lower than that

253 identified by olfactory testing. ${ }^{28}$ They also reported a discrepancy in self-reported memory

254 depending on the degree of cognitive dysfunction. ${ }^{29}$ In other words, it is difficult to screen for

255 memory loss and olfactory deficits in older people without testing, and our results suggest that

256 olfactory deficits are not easily recognized in daily life. Since olfactory identification deficits are

257 associated with an increased risk of mortality in older individuals, it is important to be aware of

258 olfactory function decline. ${ }^{30} \mathrm{~A}$ simple olfactory test, such as the UPSIT, may facilitate the

259 recognition of olfactory deficits and memory loss.

260 The strength of our study is that we performed mild cognitive dysfunction tests and olfactory

261 identification tests on older adults in Japan who had not been diagnosed with MCI or dementia.

262 Additionally it was involved a large number of older people who were not visiting local

263 hospitals. Early detection is essential for timely dementia treatment. However, in many cases,

264 some level of cognitive dysfunction is already present when the patient first visits a hospital for

265 various psychological tests. Therefore, it is necessary to screen people with undiagnosed 
266 cognitive dysfunction with tools that are easily accessible outside the hospitals to enable referral

267 to hospitals at early disease stages.

268 A limitation of this study is that the results may be subject to an age bias. However, as the MPI

269 score which sensitively reflects the distinction between normal aging and MCI by adjusting for

270 confounding factors such as age, sex, and educational background, correlated with the olfactory

271 identification score, this score was indicated to reflect not only age but also cognitive function.

272 Previous reports have shown that the effects of aging on the sense of smell are minor and

273 gradual, ${ }^{31}$ suggesting that these effects are not due to aging per se, but due to cognitive

274 dysfunction. ${ }^{32}$ Another limitation is that the number of olfactory items was limited because of the

275 time required for the test. Although several olfactory tests currently exist, none of them can be

276 used to diagnose or monitor dementia. Such a test should be specific to the condition and culture

277 of interest, ${ }^{33}$ and we await the development of a single, reliable assessment tool that can be used

278 in a short time. In addition, since the participants in this study were older adults who could

279 voluntarily participate in the event, we considered the cognitive function decline in the

280 participants to be mild. However, 20 participants with an MMSE score of $\leq 23$ were included in

281 the cognitively impaired group, suggesting that some dementia equivalents may have been

282 included. This study targeted Japanese people; however, due to the cultural background in Japan,

283 we could not conduct a survey on ethnicity; hence, at least those who live in Japan were targeted.

284 Since there were only a few elderly foreign nationals (less than 1\%) in the city surveyed in this

285 study, it is not expected to have a significant impact. Although this was a cross-sectional study,

286 many studies have shown that olfactory function can predict longitudinal changes from normal

287 cognitive function to MCI and from MCI to dementia. ${ }^{34}$ Therefore, longitudinal evaluation of 
288 cognitive function in people with olfactory deficits may help predict the onset of MCI and

289 dementia in older, community-dwelling people, and thus may be a useful screening tool.

290

\section{Conclusions}

292 We confirmed that cross-sectional olfactory identification deficits at baseline in community-

293 dwelling older adults in Japan reflected mild cognitive dysfunction and that olfactory

294 identification function was significantly correlated with cognitive function. The results suggest

295 the utility of introducing olfactory tests as screening tools for cognitive screening in health

296 checkups for older individuals in future.

297

298 Acknowledgments

299 The authors acknowledge the role of Coomin Corp in conducting this study.

300 We would also like to express our sincere gratitude to the associations in the target areas and to

301 all the people who understood the purpose of this study and willingly cooperated.

302

\section{References}

304 1. Prince M, Wimo A, Guerchet M, Ali G-C, Wu Y-T, Prina M. World Alzheimer Report

305 2015. Available from: https://www.alz.co.uk/research/WorldAlzheimerReport2015.pdf

$306 \quad$ (accessed on Nov 8, 2021)

307 2. Prince M, Comas-Herrera A, Knapp M, Guerchet M, Karagiannidou M. World

308 Alzheimer Report 2016. Available from:

309 https://www.alzint.org/u/WorldAlzheimerReport2016.pdf (accessed on Nov 8, 2021)

3103 . Callahan CM, Hendrie HC, Tierney WM. Documentation and evaluation of cognitive

311 impairment in elderly primary care patients. Ann Intern Med 1995;122:422-429.

312 4. Alves J, Petrosyan A, Magalhaes R. Olfactory dysfunction in dementia. World J Clin

313 Cases 2014;2:661-667.

314 5. Wilson RS, Schneider JA, Arnold SE, Tang Y, Boyle PA, Bennett DA. Olfactory

315 identification and incidence of mild cognitive impairment in older age. Arch Gen

316 Psychiatry 2007;64:802-808. 
317 6. Roalf DR, Moberg MJ, Turetsky BI, Brennan L, Kabadi S, Wolk DA, Moberg PJ. A

318 quantitative meta-analysis of olfactory dysfunction in mild cognitive impairment. J

$319 \quad$ Neurol Neurosurg Psychiatry 2017;88:226-232.

3207 Windon MJ, Kim SJ, Oh ES, Lin SY. Predictive value of olfactory impairment for

321 cognitive decline among cognitively normal adults. Laryngoscope 2020;130:840-847.

322 8. Makizako M, Makizako H, Doi T, Uemura K, Tsutsumimoto K, Miyaguchi H, Shimada

323 H. Olfactory identification and cognitive performance in community-dwelling older

$324 \quad$ adults with mild cognitive impairment. Chem Senses 2014;39:39-46.

325 9. Yaffe K, Freimer D, Chen H, Asao K, Rosso A, Rubin S, Tranah G, Cummings S,

326

327

328

329

330

331

332

333

334 Simonsick E. Olfaction and risk of dementia in a biracial cohort of older adults. Neurology 2017;88:456-462.

10. Liang X, Ding D, Zhao Q, Guo Q, Luo J, Hong Z. Association between olfactory identification and cognitive function in community-dwelling elderly: The Shanghai aging study. BMC Neurol 2016;16:1-9.

11. Pinto JM, Schumm LP, Wroblewski KE, Kern DW, McClintock MK. Racial disparities in olfactory loss among older adults in the United States. Journals Gerontol 2014;69:323329.

12. Shankle WR, Romney AK, Rara J, Fortier D, Dick MB, Chen JM, Chan T Sun X.

335

336

337 Methods to improve the detection of mild cognitive impairment. Proc Natl Acad Sci U S A 2005;102:4919-4924.

13. Shankle WR, Mangrola T, Chan T, Hara J. Development and validation of the Memory

338

339

340

341

342

343

344

345

346

347

348

349 Performance Index: Reducing measurement error in recall tests. Alzheimer's Dement 2009;5:295-306.

14. Cho A, Sugimura M, Nakano S, Yamada T. Current topics in management: The Japanese MCI screen for early detection of Alzheimer 's disease and related disorders. Am J Alzheimer's Dis Other Dementias 2008;23:162-166.

15. Tsoi KKF, Chan JYC, Hirai HW, Wong SYS, Kwok TCY. Cognitive tests to detect dementia a systematic review and meta-analysis. JAMA Intern Med 2015;175:14501458.

16. Doty RL, Shaman P, Kimmelman CP, Dann MS. University of Pennsylvania smell identification test: A rapid quantitative olfactory function test for the clinic.

Laryngoscope 1984;94:176-178.

17. Bohnen NI, Gedela S, Kuwabara H, Constantine GM, Mathis CA, Studenski SA, Moore

350

351

352 RY. Selective hyposmia and nigrostriatal dopaminergic denervation in Parkinson's disease. J Neurol 2007;254:84-90.

353

354

18. Woodward MR, Amrutkar CV, Shah HC, Benedict RHB, Rajakrishnan S, Doody RS, Yan L, Szigeti K. Validation of olfactory deficit as a biomarker of Alzheimer disease. Neurol Clin Pr 2017;7:5-14. 
355 19. Ogihara H, Kobayashi M, Nishida K, Kitano M, Takeuchi K. Applicability of the cross-

356

357

358

359

360

361

362

363

364

365

366

367

368

369

370

371

372

373

374

375

376

377

378

379

380

381

382

383

384

385

386

387

388

389

390

391

392

culturally modified University of Pennsylvania Smell Identification Test in a Japanese population. Am J Rhinol Allergy 2011;25:404-410.

20. Rahayel S, Frasnelli J, Joubert S. The effect of Alzheimer's disease and Parkinson's disease on olfaction: A meta-analysis. Behav Brain Res 2012;231:60-74.

21. Umeda-Kameyama Y, Ishii S, Kameyama M, Kondo K, Ochi A, Yamasoba T, Ogawa S, Akishita M. Heterogeneity of odorant identification impairment in patients with Alzheimer's disease. Sci Rep 2017;7:4798.

22. Vyhnalek M, Magerova H, Andel R, Nikolai T, Kadlecova A, Laczo J, Hort J. Olfactory identification in amnestic and non-amnestic mild cognitive impairment and its neuropsychological correlates. J Neurol Sci 2015;349:179-184.

23. Devanand DP. Olfactory identification deficits, cognitive decline, and dementia in older adults. Am J Geriatr Psychiatry 2016;24:1151-1157.

24. Masurkar AV, Devanand DP. Olfactory dysfunction in the elderly: Basic circuitry and alterations with normal aging and Alzheimer's disease. Curr Geriatr Rep 2014;3:91-100.

25. Yoshii F, Onaka H, Kohara S, Ryo M, Takahashi W. Association of smell identification deficit with Alzheimer's disease assessment scale-cognitive subscale, Japanese version scores and brain atrophy in patients with dementia. Eur Neurol 2019;81:145-151.

26. Attems J, Walker L, Jellinger KA. Olfactory bulb involvement in neurodegenerative diseases. Acta Neuropathol 2014;127:459-475.

27. Sohrabi HR, Bates KA, Weinborn MG, Johnston ANB, Bahramian A, Taddei K, Laws SM, Rodrigues M, Morici M, Howard M, Martins G, MacKay-Sim A, Gandy SE, Martins RN. Olfactory discrimination predicts cognitive decline among communitydwelling older adults. Transl Psychiatry 2012;2:e118-e117.

28. Murphy C, Schubert CR, Cruickshanks KJ, Klein BEK, Klein R, Nondahl DM. Prevalence of olfactory impairment in older adults. J Am Med Assoc 2002;288:23072312.

29. Silva MR, Moser D, Pflüger M, Pusswald G, Stögmann E, Dal-Bianco P, Auff E, Lehrner $\mathrm{J}$. Self-reported and informant-reported memory functioning and awareness in patients with mild cognitive impairment and Alzheimers disease. Neuropsychiatrie 2016;30:103112.

30. Devanand DP, Lee S, Manly J, Andrews H, Schupf N, Masurkar A, Stern Y, Mayeux R, Doty RL. Olfactory identification deficits and increased mortality in the community. Ann Neurol 2015;78:401-411.

31. Mackay-Sim A, Johnston ANB, Owen C, Burne THJ. Olfactory ability in the healthy population: Reassessing presbyosmia. Chem Senses 2006;31:763-771.

32. Finkel D, Pedersen NL, Larsson M. Olfactory functioning and cognitive abilities: A twin study. J Gerontol B Psychol Sci Soc Sci 2001;56:226-233. 
393 33. Gros A, Manera V, De March CA, Guevara N, König A, Friedman L, Robert P, 394 Golebiowski J, David R. Olfactory disturbances in ageing with and without dementia:

395 Towards new diagnostic tools. J Laryngol Otol 2017;131:572-579.

396 34. Devanand DP, Lee S, Manly J, Andrews H, Schupf N, Doty RL, Stern Y, Zahodne LB, 397 Louis ED, Mayeux R. Olfactory deficits predict cognitive decline and Alzheimer dementia in an urban community. Neurology 2015;84:182-189.

399 


\section{Table $\mathbf{1}$ (on next page)}

Characteristics of the participants

Abbreviations: MPI, Memory Performance Index; UPSIT-J, Japanese version of the University of Pennsylvania Smell Identification Test; MMSE, Mini-Mental State Examination; SD, standard deviation. 


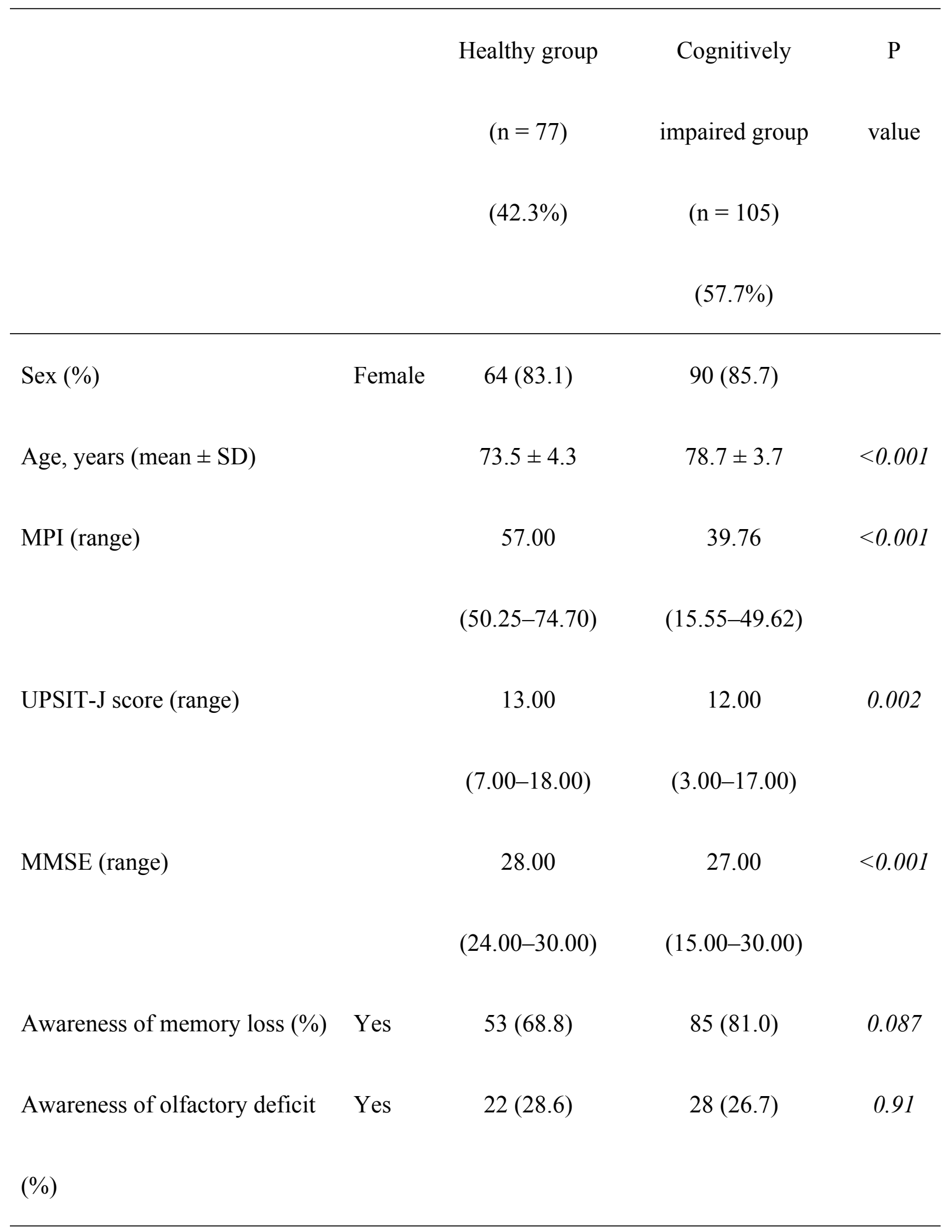


Figure 1

Differences in median scores between healthy and cognitively impaired groups

Abbreviations: UPSIT score, University of Pennsylvania Smell Identification Test Score

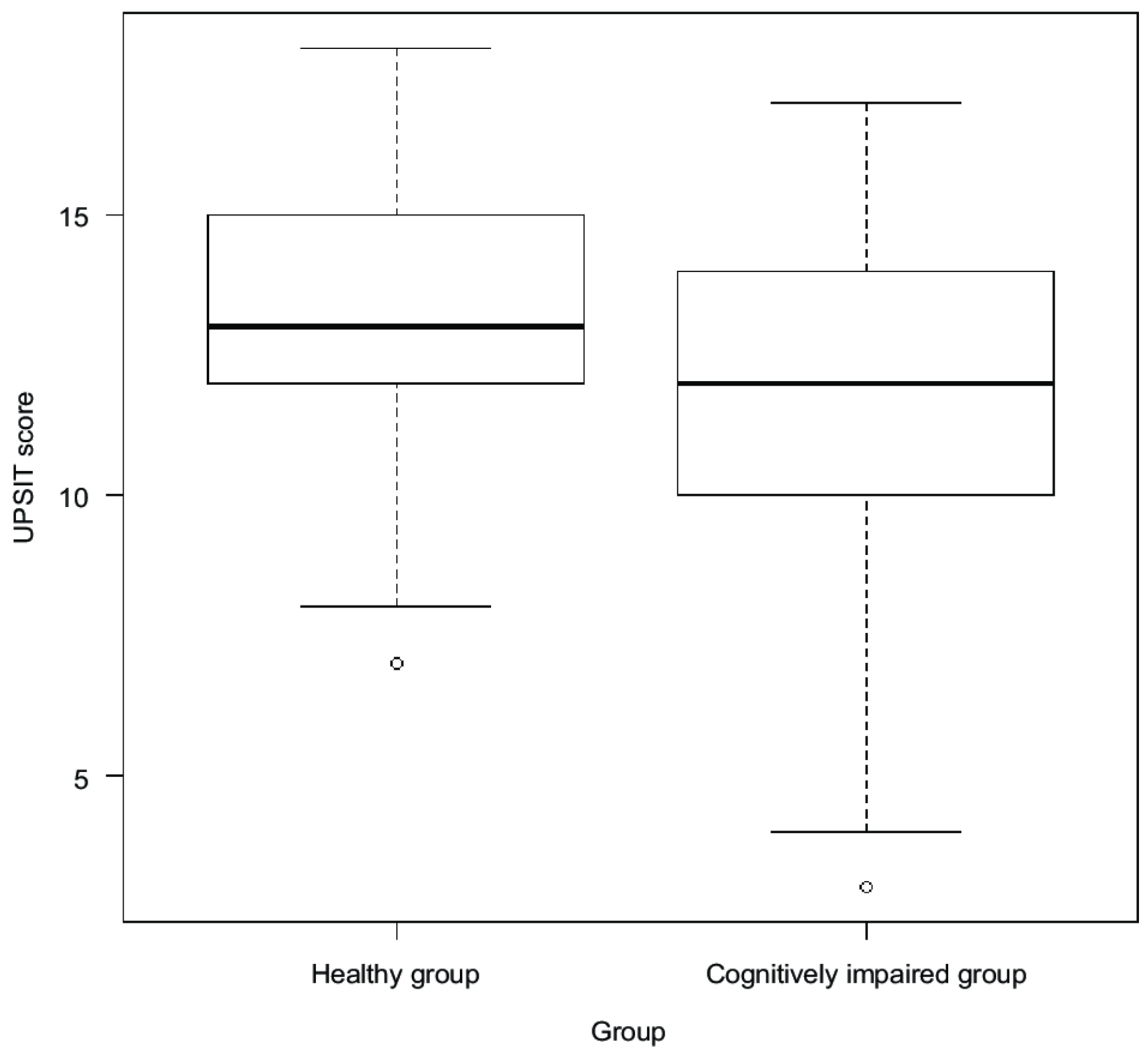


Figure 2

Correlation between MPI and UPSIT scores of all participants

Abbreviations: MPI, Memory Performance Index; UPSIT score, University of Pennsylvania Smell Identification Test Score

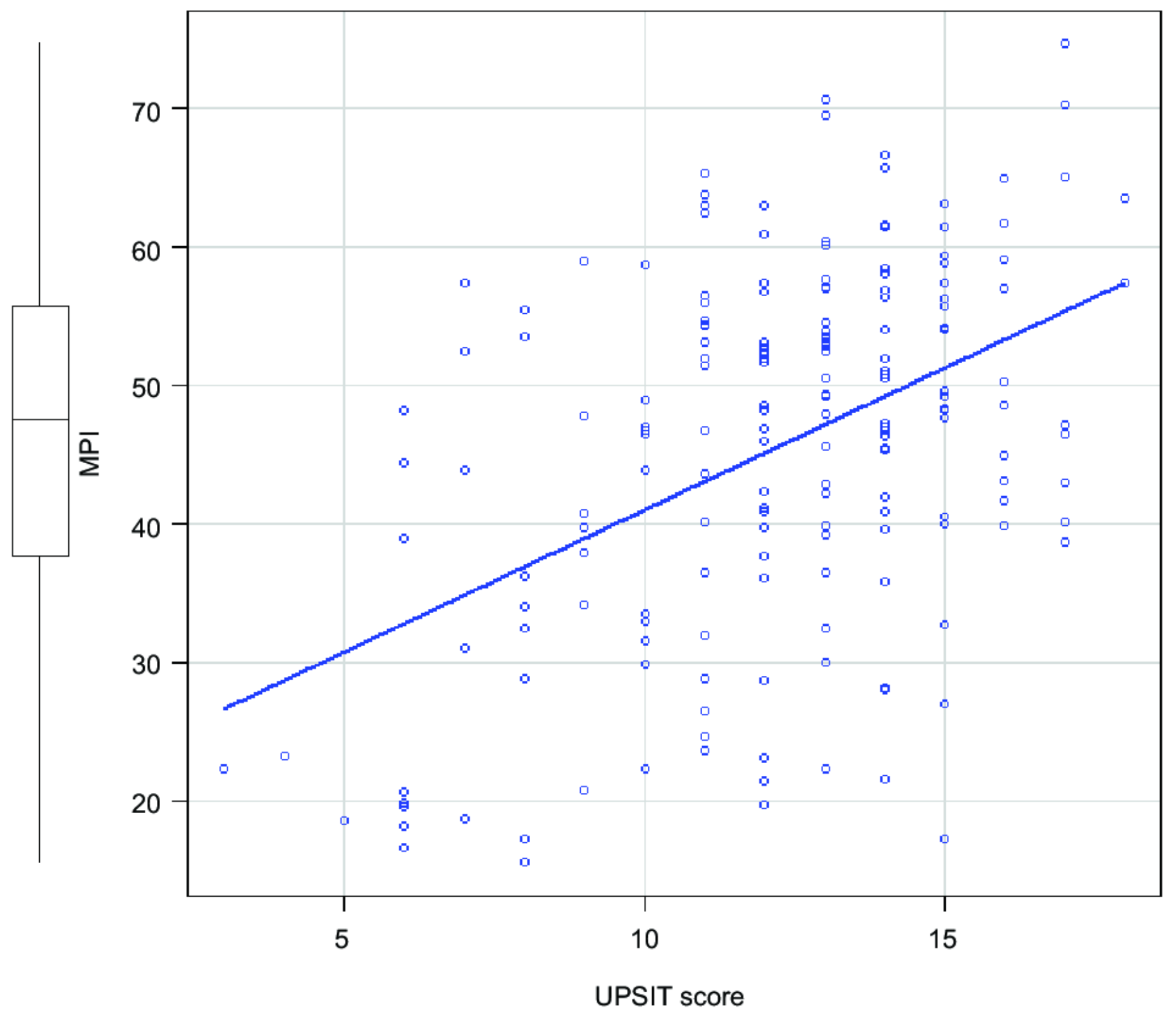

